\title{
ON THE YANG-MILLS-HIGGS EQUATIONS
}

\author{
BY CLIFFORD HENRY TAUBES ${ }^{1}$
}

I. Introduction. The purpose of this note is to announce new results for the Yang-Mills-Higgs equations on $R^{3}$. These SU(2) Yang-Mills-Higgs equations are a set of partial differential equations where the unknown is a pair, $c=$ $(A, \Phi)$, with $A$ a connection on the vector bundle $E=R^{3} \times \mathfrak{s u}(2)$ and $\Phi$ a section of $E$. Here $\mathfrak{s u}(2)=$ Lie alg. $\mathrm{SU}(2)$. These equations are

$$
D_{A} * F_{A}+\left[\Phi, D_{A} \Phi\right]=0, \quad D_{A} * D_{A} \Phi=0,
$$

with boundary condition $\lim _{|x| \rightarrow \infty}|\Phi|(x)=1$. Here, the notation follows [1]. That is, $F_{A}$ is the curvature of $A, D_{A}$ is the exterior covariant derivative on $\bigwedge T^{*} \otimes E$ and $[\cdot, \cdot]$ is the natural, graded bracket on $\bigwedge T^{*} \otimes E:$ If $\omega, \eta$ are, respectively, $E$-valued $p, q$ forms, then $[\omega, \eta]=\omega \wedge \eta-(-1)^{p q} \eta \wedge \omega$. The $*$ in (1) is the Hodge star on $\bigwedge T^{*}$ from the Euclidean metric on $T^{*}$. The norm $|\cdot|$ on $T^{*} \otimes E$;is that induced from the Euclidean metric on $T^{*}$ and the Killing metric on $\mathfrak{s u}(2)$.

Equation (1) is the variational equation of an action functional

$$
A(A, \Phi)=\frac{1}{2} \int_{R^{3}}\left\{\left|F_{A}\right|^{2}(x)+\left|D_{A} \Phi\right|^{2}(x)\right\} d^{3} x .
$$

One is to consider $A$ as a function on the set

$$
C=\left\{\operatorname{smooth}(A, \Phi): A(A, \Phi)<\infty \text { and } 1-|\Phi|(x) \in L^{6}\left(R^{3}\right)\right\} .
$$

$C$ is topologized as follows [2]: Let $\theta$ denote the flat, product connection on $E$. The topology of $C$ is defined to be the weakest for which the map sending $C=(A, \Phi) \in C$ to

$$
(A-\theta, A(c)) \in \Gamma\left(T^{*} \otimes E\right) \times \Gamma(E) \times[0, \infty)
$$

is continuous.

The topological group

$$
\begin{aligned}
\mathcal{G} & =\{\text { smooth, unitary automorphisms of } E\}, \\
& =C^{\infty}\left(R^{3} ; \mathrm{SU}(2)\right) /\{ \pm 1\}
\end{aligned}
$$

acts continuously on $C$ and leaves $A$ and (1) invariant. The subgroup $\mathcal{G}_{0}=$ $\{g \in \mathcal{G}: g(0)=1\}$ acts freely on $C$. Let $B=C / \mathcal{G}_{0}$ denote the quotient. The functional $A$ descends as a continuous, $\mathrm{SO}(3)$ invariant function on $B$.

The relationship between $A$ and $B$ is described in the following theorems.

Received by the editors October 28, 1983.

1980 Mathematics Subject Classification. Primary 35Q20, 58G20, 81E10; Secondary 55Q52.

${ }^{1}$ NSF Postdoctoral Fellow in Mathematics 
THEOREM 1. The space $B$ is homotopic to the space of smooth maps from $S^{2}$ to $S^{2}, \operatorname{Maps}\left(S^{2} ; S^{2}\right)$. The space B has a compatible Fréchet space structure for which $A$ is a smooth function. With this smooth structure, a point $[A, \Phi] \in B$ is a critical point of $\mathcal{A}$ if and only if $(A, \Phi) \in C$ satisfies (1).

Thus, $B$ is topologically the disjoint union of spaces $B_{n}, n \in Z$, with each $B_{n}$ homotopic to the space of degree $n$ maps from $S^{2}$ to $S^{2}$ (cf. [2, §3 and Appendix 2]).

It has previously been established that $A$ takes on its minimum on each $B_{n}$ $[3,4,5]$, and, by a min-max argument, that $A$ has a nonminimal critical point on $B_{0}[2,6]$. A new min-max theory for $A$ on $B$ and the nontrivial topology of $B_{n}$ results in

Theorem 2. For each $n, A$, when restricted to $B_{n}$, has an unbounded set of critical values.

Restrict $A$ to $B_{n}, n \in Z$. One observes that $A \geq 4 \pi|n|$, with equality at the minima on $B_{n}$ where the Bogomol'yni equations are satisfied [7]:

$$
F_{A}=\operatorname{sign}(n) * D_{A} \Phi .
$$

The set of $[A, \Phi] \in B_{n}$ which solve (5) is called the moduli space, $\mathcal{M}_{n}$, of selfdual monopoles. One can study these spaces using twistor techniques $[8,9$, 10]. But still, little is known about them.

By applying the new min-max theory as in [11], one can study the topology of $\mathcal{M}_{n}$ via the embedding of $\mathcal{M}_{n}$ in $B_{n}$. Of import here is an observation from [12] that the hessian of $A$ at a nonminimal critical point in $B_{n}$ must have index larger than $|n|$. One obtains as a result

THEOREM 3. For each $n$, the inclusion $\mathcal{M}_{n} \rightarrow B_{n}$ induces an isomorphism of the pointed homotopy groups $\pi_{l}(\cdot)$ for $0 \leq l \leq|n|-1$ and an epimorphism of $\pi_{|n|}(\cdot)$.

The details and proofs of these results are forthcoming [13]. The motivation for the new min-max theory for $A$ is obtained by considering the following isotopy of $B$ : First, flow from $c=[A, \Phi]$ along a vector field which is minus the gradient of $A$ on domains in $R^{3}$ where $\Psi \equiv\left(\left|F_{A}\right|,\left|D_{A} \Phi\right|, 1-|\Phi|\right)$ is small, and which is zero elsewhere. This flow converges since it is a perturbation of a linear flow. The limit, $c^{\prime}=\left[A^{\prime}, \Phi^{\prime}\right]$ satisfies (1) on domains in $R^{3}$ where $\Psi$ is small. Next, flow along minus the gradient flow of $\mathcal{A}$, projected to maintain (1) in the small $\Psi$ domains. The a priori estimates from [1] for $\Psi$ in the small $\Psi$ regions (where (1) is satisfied) allow one to prove that the large $\Psi$ regions remain in a bounded set on $R^{3}$ for all $t \in(0, \infty)$. Under these conditions, $\mathrm{K}$. Uhlenbeck's compactness theorems [14] imply convergence for the flow.

\section{REFERENCES}

1. A. Jaffe and C. H. Taubes, Vortices and monopoles, Birkhauser, 1980.

2. C. H. Taubes, The existence of a non-minimal solution to the SU(2) Yang-Mills-Higgs equations on $R^{3}$. I, II, Comm. Math. Phys. 86 (1982), 257; ibid. Comm. Math. Phys. 86 (1982), 299. 
3. _ The existence of multi-monopole solutions to the non-abelian, Yang-Mills-Higgs equations for arbitrary, simple gauge groups, Comm. Math. Phys. 80 (1981), 343.

4. R. Ward, A Yang-Mills-Higgs monopole of charge 2, Comm. Math. Phys. 80 (1981), 137.

5. E. Corrigan and P. Goddard, An n-monopole with $4 n-1$ degrees of freedom, Comm. Math. Phys. 80 (1981), 575.

6. D. Groisser, SU(2) Yang-Mills theory on $R^{3}$, Harvard Univ. Preprint, 1983.

7. E. B. Bogomol'nyi, The stability of classical solutions, Soviet J. Nuclear Phys. 24, (1976), 449.

8. N. J. Hitchin, Monopoles and geodesics, Comm. Math. Phys. 83 (1982), 579.

9. W. Nahm, All self-dual monopoles for arbitrary gauge group, TH. 3172-CERN, 1981 (preprint).

10. N. J. Hitchin, On the construction of monopoles, Comm. Math. Phys. 89 (1983), 145.

11. C. H. Taubes, Path connected Yang-Mills moduli spaces (preprint).

12. __ Stability in Yang-Mills theories, Comm. Math. Phys. (to appear).

13. ___ A min-max theory for the Yang-Mills-Higgs equations (forthcoming).

14. K. K. Uhlenbeck, Connections with $L^{p}$ bounds on curvatures, Comm. Math. Phys. 83 (1982), 31 .

Department of Mathematics, University of California, Berkeley, CaliFORNIA 94720 